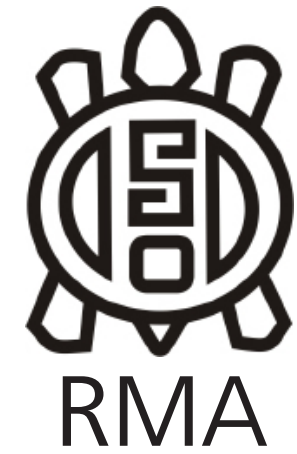

Dossier

\section{Un espacio doméstico de fines del primer milenio en El Colorado, sur de Yocavil, Catamarca, Argentina. Primeros resultados de las investigaciones de campo}

\author{
A domestic space of the end of the first millennium in El Colorado, \\ south of Yocavil, Catamarca, Argentina. First results of field research
}

Valeria Palamarczuk*, Cecilia B. Raíces Montero**,Natalia Petrucci***, Catriel

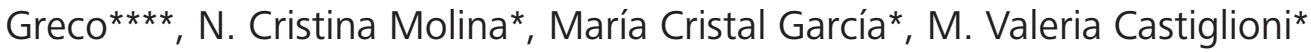

"Instituto de las Culturas (IDECU), UBA-CONICET, Museo Etnográfico "Juan B. Ambrosetti". E-mail:valepala@yahoo.com.ar; cristinamol59@hotmail.com; mariacristalgarcia@hotmail.com; valeriacastiglioni@gmail.com

\begin{abstract}
** Banco Nacional de Datos Genéticos, Instituto de las Culturas (IDECU), UBA-CONICET, Museo Etnográfico "Juan B. Ambrosetti". E-mail: cecirai@hotmail.com

${ }^{* * *}$ Laboratorio de Etnobotánica y Botánica Aplicada (LEBA), Facultad de Ciencias Naturales y Museo, Universidad Nacional de La Plata, Argentina. E-mail: nataliapetrucci@fcnym.unlp.edu.ar

${ }^{\star \star \star \star}$ CONICET - Departamento de Geología, Universidad Nacional de San Luis. E-mail: catrielgreco@gmail.com
\end{abstract}

\begin{abstract}
Resumen
Este trabajo aporta a la elaboración de una imagen sobre los ámbitos domésticos construidos y habitados durante los últimos siglos del primer milenio D.C en Yocavil, una época para la que existe escaso registro en la actualidad. Se presenta una síntesis de las tres últimas temporadas de campo, efectuadas en 2016, 2017 y 2018, en la localidad arqueológica El Colorado, sur de Yocavil, provincia de Catamarca, Argentina. Las actividades se centraron en la indagación de una unidad residencial "Detección 28" compuesta por dos recintos (Estructura 65 y Estructura 66), situada en el Sector Conoide Norte de la localidad. Las excavaciones en área permitieron detectar una ocupación en la E 65, en la cual se alcanzaron depósitos estériles, y un mínimo de dos ocupaciones en la E 66, cuya excavación aún no ha concluido. Considerando los resultados de un fechado radiocarbónico y las características estilísticas de la arquitectura y de los materiales recuperados en las superficies de uso, se estableció que la E65 y la ocupación superior de la E66 corresponden a los últimos siglos del primer milenio de la era. Se presentarán detalles de la arquitectura y tendencias observadas en los conjuntos cerámico y lítico recuperados.
\end{abstract}

Palabras clave: Valle de Yocavil; Período Formativo; Período Medio; Espacio doméstico

\begin{abstract}
This work contributes to the elaboration of an image about the domestic areas built and inhabited during the last centuries of the first millennium A.D. in Yocavil, a time for which there is currently little record. We present a synthesis of the last three field seasons, carried out in 2016, 2017 and 2018, in the archaeological town of El Colorado, south of Yocavil, province of Catamarca, Argentina. The activities focused on the investigation of a residential unit "Detection 28" composed of two enclosures (Structure E65 and Structure E66), located in the North Conoid Sector of the locality. Excavations in the area allowed to detect an occupation in E65, in which sterile deposits were reached, and a minimum of two occupations in E66, whose excavation has not yet been completed. Considering the results of a radiocarbon dating and the stylistic characteristics of the architecture and materials recovered in the use surfaces, it was established that E65 and the superior occupation of E66 correspond to the last centuries of the first millennium of the era. Details of the architecture, and advances in the analysis of the ceramic and lithic set will be presented.
\end{abstract}

Keywords: Yocavil valley; Formative Period; Middle Period; Household 
La localidad arqueológica El Colorado, ubicada en el suroeste del valle de Yocavil, es objeto de nuestras investigaciones desde el año 2010. El registro material de diferentes momentos de la historia en este sector indica que se trata de un espacio que fue habitado a través de un tiempo largo; hay evidencias de diversas instalaciones de pequeña escala que abarcan temporalmente desde el primer milenio de la era hasta fines del siglo XX (Palamarczuk 2016). Algunas de las instalaciones fueron reocupadas por siglos, otras presentan usos temporalmente más discretos. En conjunto representan una valiosa configuración material para el estudio del uso del espacio y de la organización de los ámbitos residenciales de pequeña escala a lo largo de una historia compleja. En este proceso se sucedieron diferentes sistemas de organización social y las poblaciones locales se articularon con organizaciones político económicas de mayor escala, son ejemplos el desarrollo de centros poblados conglomerados cercanos, el proceso de expansión inca, la colonización europea y la organización del estado republicano. Nos proponemos avanzar en esos campos a través de la distinción de las ocupaciones, de la reconfiguración y del abandono de sitios, según una perspectiva que articula las distintas temporalidades de los procesos históricos (Braudel 1970).

En el norte de la localidad (Sectores Norte y Conoide Norte) se concentra la mayor cantidad de evidencia de época temprana: fragmentos de alfarería y arquitectura. Debido a los procesos erosivos que operan en los abanicos aluviales o conoides (Maldonado et al. 2012) las señales de las antiguas ocupaciones formativas en el Sector Conoide Norte son visibles en la superficie actual; los sedimentos no cubrieron por completo las bases de piedra de las estructuras y los usos posteriores del espacio no borraron su señal.

Inicialmente el trabajo en este sector se enmarcó en nuestro objetivo de detectar ocupaciones residenciales de inicios del segundo milenio. Planteamos como hipótesis, que espacios habitacionales construidos de acuerdo con modalidades arquitectónicas más antiguas -enmarcadas temporalmente en sentido amplio en el primer milenio-, pudieron ser reocupados, de modo continuo o discontinuo, en momentos posteriores ${ }^{1}$.

Las actividades se centraron en la indagación de una unidad residencial a la que denominamos "Detección 28" situada en el Sector Conoide Norte, en el extremo suroriental de la geoforma, con un área aproximada de $600 \mathrm{~m}^{2}$. Está compuesta, según el registro de superficie, por un mínimo de dos recintos (Estructuras 65 y 66), separados entre sí por un espacio de 4 metros, y una estructura de contención construida en piedra sobre el quiebre de pendiente de una cárcava que discurre hacia el SE (Figura 1). La selección del área a estudiar se fundó en la observación en superficie de arquitectura pretardía (dos recintos ortogonales con muros de cimientos de piedra simples y dobles) y de fragmentos cerámicos de estilos indicativos de fines del primer milenio

\footnotetext{
1 Este planteo se basa en nuestra experiencia de trabajo en otro conjunto arquitectónico de la localidad (Detección 2), ubicado en el Sector Norte. Allí, en un recinto con arquitectura típica del Período Tardío (Estructura 3) las excavaciones definieron ocupaciones sucesivas de época Tardía, Inca y Colonial Temprana (Palamarczuk et al. 2016).
}

D.C -gris pulido, gris pulido con incisiones, alisado ordinario ante pulido, Guachipas (sensu Serrano 1958)-, y de inicios del Período Tardío -alisadas con pasta oxidante afines a San José, peinado ordinario-. La presencia de estos últimos estilos cerámicos en el conjunto de superficie se tomó como un posible indicador de ocupación tardía de los recintos con arquitectura temprana.

\section{Pasos de la investigación de campo}

El trabajo de campo se realizó a lo largo de tres temporadas (abril de 2016, 2017 y 2018) e incluyó las siguientes actividades: a) recolección de materiales de superficie, b) desmalezamiento del espacio con arquitectura, c) relevamiento con drone y elaboración de fotogrametría del sector, d) relevamiento topográfico de superficie, e) realización de un sondeo estratigráfico en la Estructura 65, f) excavación en área extendida de espacios intramuros y extramuros en las Estructuras 65 y 66 de acuerdo con el método estratigráfico, g) elaboración de plantas de superficie y de cada decapado mediante fotogrametría y relevamiento topográfico, h) procesamiento por técnica de flotación del $10 \%$ de los sedimentos excavados en cada unidad estratigráfica y de la totalidad del sedimento comprendido en áreas de combustión.

\section{Excavaciones en $E 65$}

La Estructura 65 es un recinto rectangular con cimientos de piedra, su eje mayor tiene una orientación NE/SO de aproximadamente $45^{\circ}$, con un largo máximo interno variable de entre 3,06 y de $3,40 \mathrm{~m}$ y un ancho interno variable de entre 2,50 y 2,80 m, con una superficie intramuros de 8,6 $\mathrm{m}^{2}$. El espacio intramuros se excavó en su totalidad siguiendo el método estratigráfico hasta alcanzar el sedimento estéril, y también se excavó el espacio extramuros adyacente en los cuadrantes NO y SO.

Los cimientos de piedra de los muros afloran en la superficie actual del terreno, en su construcción se alternaron esquistos y granitos, generando un juego cromático. Los muros NE, SE y SO son de aparejo simple, están construidos con cimientos de piedras prismáticas seleccionadas, de dimensiones medianas y grandes, clavadas en el sedimento, con su eje mayor dispuesto verticalmente. Por encima de estas piedras se observan relictos de una hilada de piedras con su eje mayor dispuesto de modo horizontal. El ancho de muro resultante oscila entre 25 y $30 \mathrm{~cm}$. El muro NO se diferencia por estar construido con piedras medianas y pequeñas, con un ancho que ronda los $15 \mathrm{~cm}$. En la parte sur de este muro se identificó la abertura de acceso al recinto. A ambos lados de ésta se disponen sendas piedras de forma prismática y de mayores dimensiones que delimitan el acceso; hacia el norte es una gran piedra granítica, y hacia el sur es una laja. Se observaron piedras correspondientes a derrumbe, principalmente entre los decapados 1 a 3, tanto hacia el interior, como hacia el exterior; a juzgar por su cantidad, se puede inferir que los muros eran originalmente bajos. Es posible que sobre una base de piedras se erigiera una estructura superior de material liviano (ramas, barro) ${ }^{2}$.

\footnotetext{
${ }^{2}$ No obstante, no se puede descartar la posibilidad de que los muros fueran desmantelados luego de su abandono para construir nuevos
} 

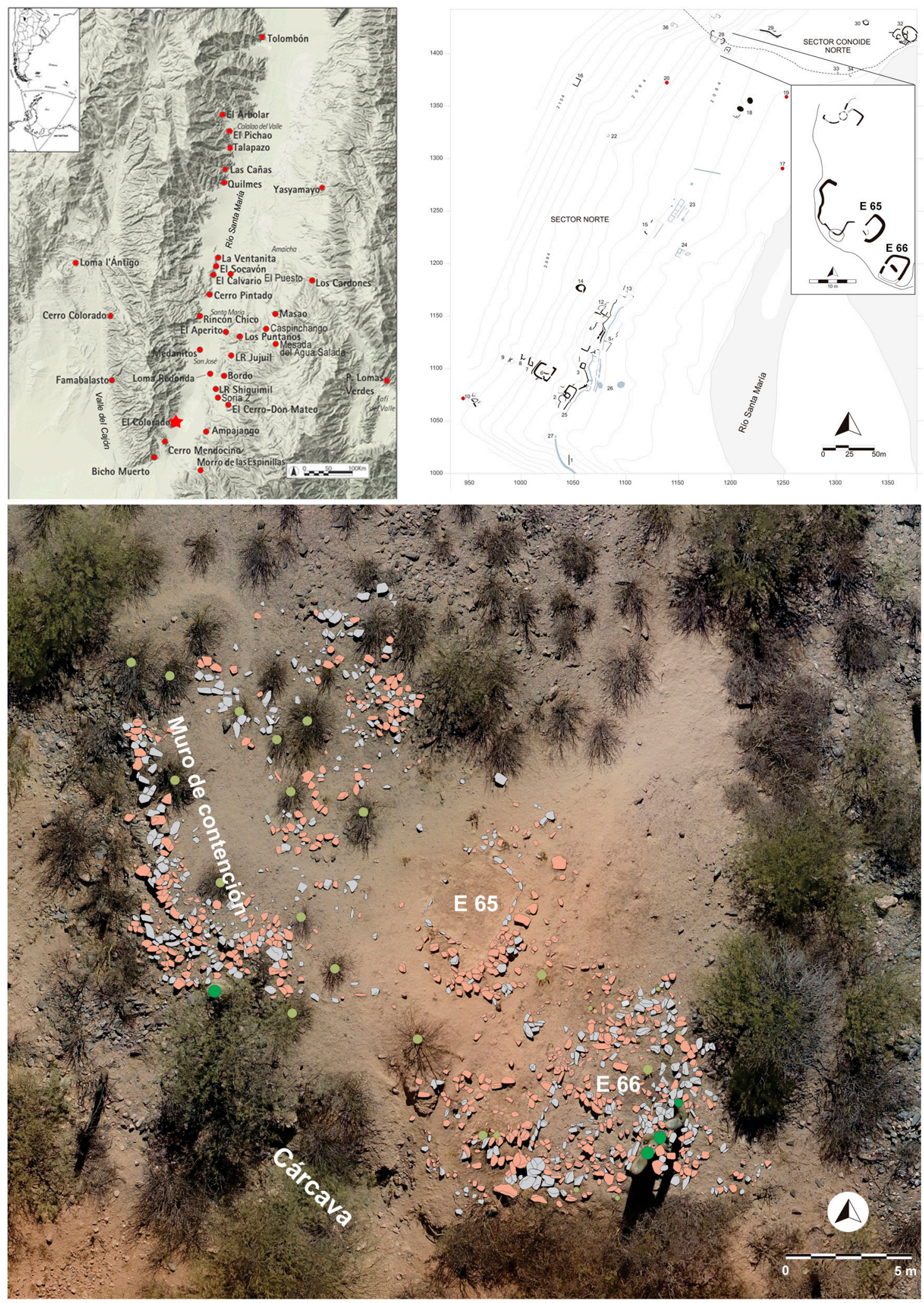

Figura 1. Izquierda: Ubicación de El Colorado en relación con otros sitios arqueológicos del Valle de Yocavil. Derecha: Planimetría de los Sectores Norte y Conoide Norte y detalle de la Detección 28. Las líneas grises indican construcciones subactuales. Los puntos rojos indican posibles espacios funerarios. Abajo: Ortomosaico de la Detección 28 a partir de fotografías tomadas con drone, programa PhotoScan, abril de 2017.

Figure 1. Left: Location of El Colorado in relation to other archaeological sites in the Yocavil Valley. Right: Planimetry of North Sector and North Conoid Sector, and detail of Detection 28. Gray lines indicate subactual constructions. Red dots indicate possible burial spaces. Below: Orthomosaic of Detection 28 from drone photographs, PhotoScan program, April 2017. 
Los decapados 1 a 3 corresponden al relleno sedimentario post abandono. Al comenzar el decapado 4, a una profundidad de entre 25 y $30 \mathrm{~cm}$ con respecto a la superficie actual, se observan indicios de la cumbre de ocupación; aumenta la frecuencia y tamaño de los fragmentos cerámicos, en su mayoría dispuestos horizontalmente y se identifican algunas manchas carbonosas en el sector Este. Los decapados 4 y 5 -que se diferencian a partir de una superficie más compacta-, representan en conjunto el piso de ocupación, con una potencia que varía entre $22 \mathrm{~cm}$ hacia el norte y $33 \mathrm{~cm}$ hacia el sur. El fogón principal (Rasgo 1), ubicado al SE, posee una forma elíptica, se destaca por su tamaño $(90 \mathrm{~cm} \times 50 \mathrm{~cm})$ y potencia $(14 \mathrm{~cm})$ de otras manchas carbonosas menores. Posee un contorno neto y hacia el este el límite se refuerza con cuatro piedras medianas y una grande tiznadas. Se recuperaron en este sector semillas carbonizadas de poroto y maíz, abundantes espículas de carbón, óseo faunístico y cerámica ordinaria tiznada ${ }^{3}$. Se realizó un fechado radiocarbónico por AMS sobre una muestra de semilla de poroto -Phaseolus vulgaris- que arrojó un resultado de $1241 \pm 20$ años ${ }^{14} \mathrm{C}$ AP (AA111229) 771 a 888 cal D.C. (rango años calend. 95\%) (Bronk Ramsey 2017) y la curva de calibración del Hemisferio Sur SHCal13 (Hogg et al. 2013).

En el sector centro-oeste del recinto se descubrió una "caja" cuadrangular construida con piedras lajas medianas y pequeñas, de $50 \mathrm{~cm}$ de lado (con un interior de $40 \mathrm{x}$ $36 \mathrm{~cm}$ ) y $15 \mathrm{~cm}$ de profundidad, que mantiene la misma orientación que el recinto. Esta estructura (Rasgo 2) estaba colmatada de un sedimento blanquecino compacto que desbordaba hacia el exterior. En el fondo del rasgo se observaron sectores con sedimentos de coloraciones rojiza y grisácea. Se realizó un análisis por Difracción de Rayos X sobre una muestra del sedimento blanquecino compacto. Los resultados indican que está compuesto por abundante calcita, cuarzo, feldespatos, cristobalita y halloisita ${ }^{4}$. La excavación estratigráfica permitió corroborar la existencia de una única fase de ocupación de este recinto. Los decapados 6 y 7 son inferiores a la ocupación, pero el sedimento aún es fértil en hallazgos y espículas de carbón migrados desde estratos superiores que disminuyen progresivamente.

Entre los materiales recuperados se cuentan 138 fragmentos cerámicos de pastas gruesas (ordinario alisado) y de pastas medias a finas (gris pulido, con y sin incisiones), 12 artefactos líticos sobre andesita y cuarzo, escasos fragmentos óseos faunísticos, abundantes restos vegetales carbonizados. No se detectaron agujeros de poste; se propone que estos pudieron ubicarse en las esquinas de los muros, hacia el exterior y que la estructura estaba abierta hacia el oeste (Figura 2).

\footnotetext{
edificios en otros sectores de la localidad.

3 Con excepción de una muestra de sedimento reservada, todo el sedimento removido de este rasgo (88 litros) se procesó mediante técnica de flotación con el fin de maximizar la recuperación de macrorrestos vegetales.

${ }^{4}$ Análisis realizado por la Dra. María Cecilia Fuertes, Gerencia Química \& Instituto de Nanociencia y Nanotecnología (INN), Centro Atómico Constituyentes-CNEA, con un equipo PANalytical Empyrean, con radiación de Cobre $\mathrm{K} \alpha$, en geometría Bragg-Brentano.
}

\section{Excavaciones en E66}

La Estructura 66 se emplaza a una distancia de 4 metros al SE de la E65. Es un recinto rectangular de arquitectura muy cuidada con cimientos de piedra, su eje mayor tiene una orientación NE/SO de aproximadamente $30^{\circ}$, con un largo interno estimado de 5,50 $\mathrm{m}$ y un ancho interno máximo de $4 \mathrm{~m}$, con una superficie intramuros aproximada de $22 \mathrm{~m}^{2}$. La excavación se planteó en área extendida, cubriendo la totalidad del espacio interno y sectores extramuros hacia el norte y el oeste, buscando definir los muros tapados por derrumbe y sedimentos.

Los muros son de piedra en su parte inferior, de $60 \mathrm{~cm}$ de ancho, están conformados por un aparejo doble, con relleno de sedimentos finos. Las piedras empleadas son esquistos y granito locales, acomodados de modo que sus cantos planos se disponen sobre el borde del muro, el cual posee un aspecto muy prolijo. La hilada inferior, que constituye los cimientos, está compuesta por piedras medianas y grandes con su eje mayor vertical. Por encima de estas, la construcción continuó con piedras acomodadas con su eje mayor dispuesto horizontalmente, de las cuales se conservan hasta dos hiladas superpuestas en la parte norte del recinto, sector donde se pudieron observar con claridad las esquinas a $90^{\circ}$. Hacia el centro del recinto, con una orientación NO-SE se observó en superficie un arreglo lineal de piedras, que interpretamos como una columna derrumbada, originalmente alzada sobre el muro Oeste. La excavación expuso piedras derrumbadas de los muros, no obstante, estas no son muy abundantes, es factible que la edificación continuara elevándose con barro y otros materiales perecibles, estructurados con columnas de piedra.

El muro sur se encuentra derrumbado por el avance erosivo de una cárcava que delimita por el sur al conoide, de modo que este no pudo definirse con precisión. Se propone que la abertura del recinto se encontraba en ese muro. La superficie actual del terreno posee un buzamiento moderado hacia el suroeste, generado por la acción erosiva de la cárcava. Por tal motivo el sedimento que conforma los pisos y el relleno post abandono que colmataba la estructura se ha erosionado con mayor intensidad hacia la mitad sur de la estructura.

Los decapados 1 a 3 corresponden a derrumbe y relleno sedimentario post abandono (30,5 cm de potencia máxima). Al finalizar el decapado 3 se observan diferentes indicios compatibles con la cumbre de la ocupación; aumenta la cantidad y tamaño de los fragmentos cerámicos, en su mayoría dispuestos horizontalmente. Se identifican algunas manchas carbonosas. Los decapados 4, 5 y 6 corresponden a un piso de ocupación (potencia máxima: $15 \mathrm{~cm}$ ). El fin del decapado 6 corresponde a una acumulación de sedimentos transicional a un piso inferior netamente definido por una superficie muy compacta que se expuso en su totalidad y cuya excavación queda pendiente.

Se identificó una estructura para calzar un poste, conformada por cuatro piedras clavadas y un agujero cilíndrico (Rasgo $1)^{5}$, su ubicación es equidistante a los muros mayores, y

\footnotetext{
${ }^{5}$ Las piedras se observaban desde la superficie actual, mientras que la boca del orificio cilíndrico se abre al fin del decapado 6, por lo que se
} 

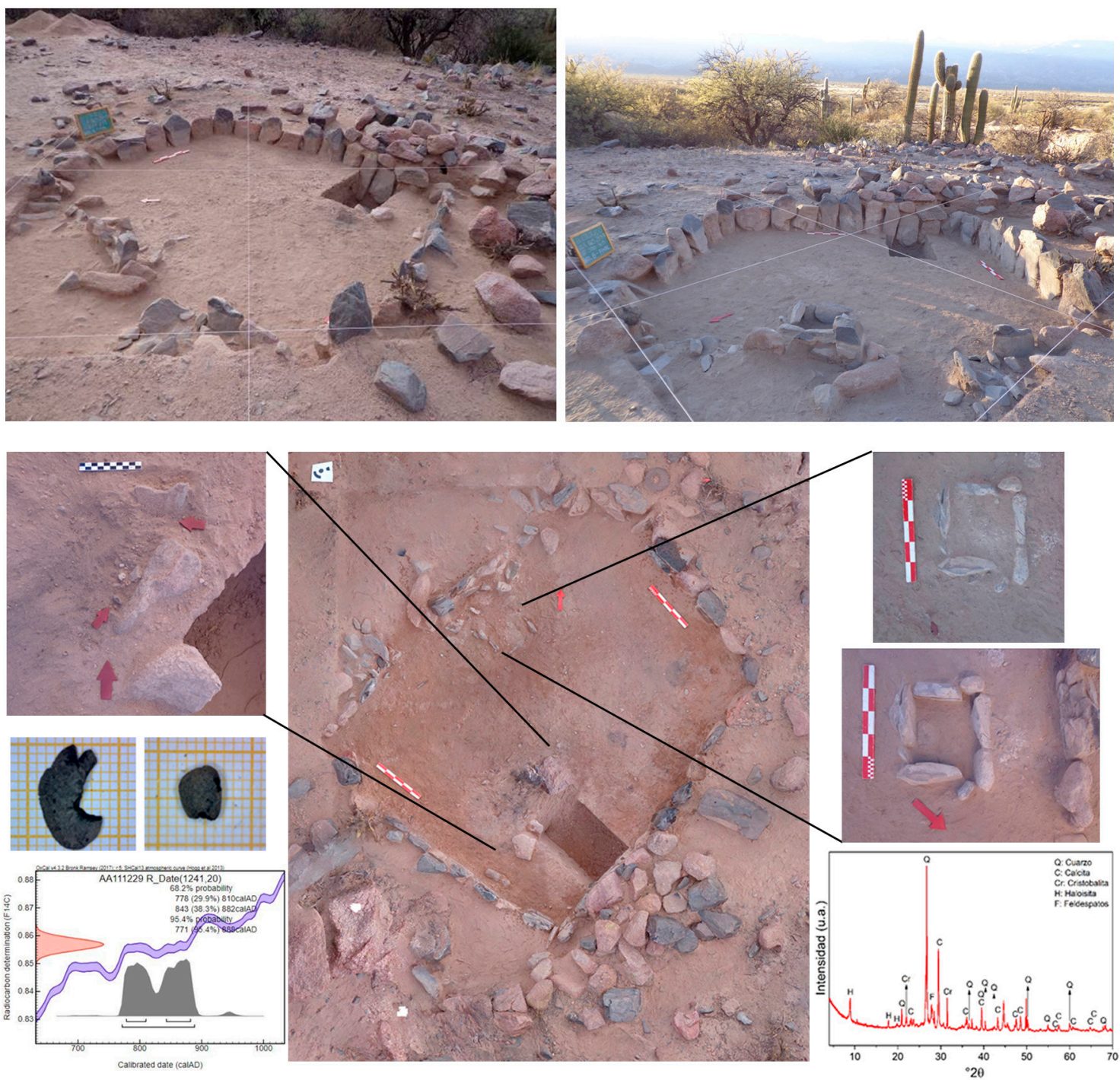

Figura 2. Vistas de la excavación en área extendida en la E65 y modelo fotogramétrico del Decapado 4 (PhotoScan). Detalles de área de combustión (Rasgo 1) y semillas fechadas por radiocarbono, y detalles de estructura para guardado de sedimentos (Rasgo 2) y difractograma del sedimento contenido.

Figure 2. Views of the excavation in open area in $E 65$ and photogrammetric model of Stratigraphic Level 4 (PhotoScan). Details of combustion area (Feature 1) and seeds dated by radiocarbon, and details of structure for sediment storage (Feature 2) and diffractogram of content sediment.

más cercana al muro sur. Se registraron dos estructuras de combustión hacia el norte del recinto, entre los decapados 4 a 6 (Rasgos 2 y 4). El Rasgo 2 se define por la presencia de dos piedras planas tipo esquisto y sedimentos carbonosos, que se aprecian en el decapado 4 (50 x $50 \mathrm{~cm}$ y $8 \mathrm{~cm}$ de potencia). El Rasgo 4 es una estructura de tipo cubeta de mayor potencia que el anterior (decapados 4 a 6), conformada por sedimentos cenicientos y carbonosos contra el muro norte con material óseo faunístico en su entorno $(35 \mathrm{~cm} \times 35 \mathrm{~cm}$ máximo y $17,6 \mathrm{~cm}$ de potencia). Una tercera estructura de combustión de mayores dimensiones se descubrió en la superficie que conforma el decapado 7 (Rasgo 5, aún no excavado), contra el muro oeste.

plantea que la ubicación del poste se mantuvo funcional en relación a las dos superficies de piso detectadas.
Por debajo de la posible columna derrumbada se halló una olla ordinaria alisada fragmentada in situ (Rasgo 3). Entre los materiales recuperados se cuentan 800 fragmentos cerámicos de pastas gruesas (ordinario alisado, ordinario peinado o marleado) y de pastas medias a finas (gris pulido, con y sin incisiones, Guachipas), 549 artefactos líticos sobre andesita y cuarzo, numerosos fragmentos óseos faunísticos -algunos carbonizados-, abundantes restos vegetales carbonizados (Figura 3).

Se plantea la existencia de al menos dos fases de ocupación de este recinto, la fase de ocupación más moderna sería coetánea a la ocupación de la Estructura 65, si bien esta apreciación deberá fundamentarse mediante análisis radiocarbónico de restos orgánicos. Los estilos alfareros recuperados sitúan a esta ocupación hacia finales del primer 

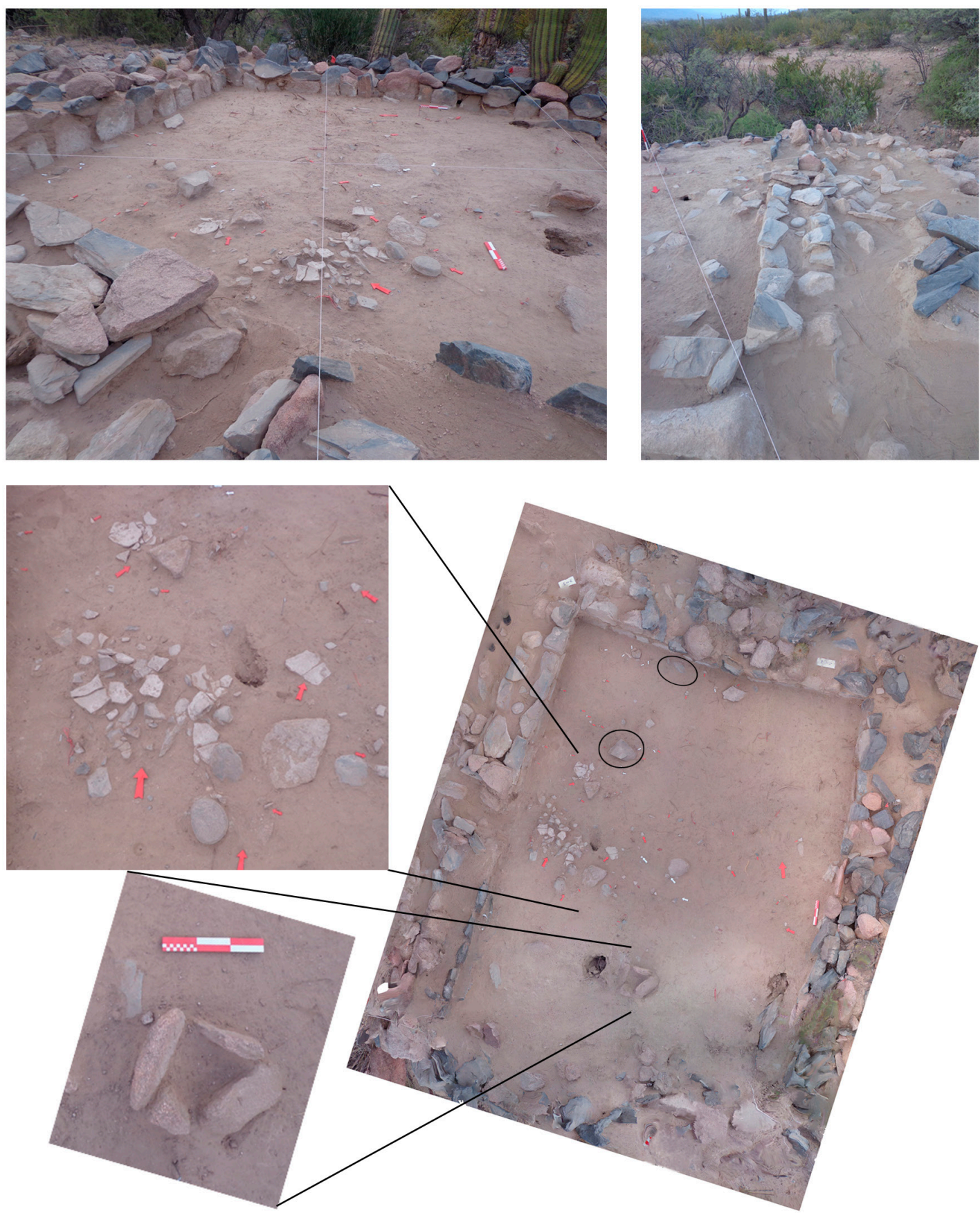

Figura 3. Vistas de la excavación en área extendida en la E66 y modelo fotogramétrico del Decapado 6 (PhotoScan). Se indican con óvalos dos áreas de combustión (Rasgos 2 y 4). Detalles de estructura de poste (Rasgo 1), olla ordinaria rota in situ (Rasgo 3).

Figure 3. Views of the excavation in open area on E66 and photogrammetric model of Stratigraphic Level 6 (PhotoScan). Two combustion areas are indicated with line ovals (Features 2 and 4). Pole structure details (Trait 1), ordinary pot broken in place (Trait 3).

milenio de la era. La fase de ocupación más antigua se determinó a partir de la superficie neta y compacta, aún pendiente de excavar. En esta superficie se definió una gran área de combustión ubicada contra la columna, y por debajo de la olla fragmentada in situ, separada de ésta por varios centímetros de sedimento depositados luego del desuso del fogón. Esta sucesión de rasgos localizados en un mismo punto, contiguo a la columna de piedra, sugiere que ésta pudo operar no solo como sostén de una estructura de techo, sino como un elemento estructurante del espacio habitado.

\section{Reconociendo los signos de época para una historia local}

Si bien nuestra hipótesis de trabajo inicial no se confirmó, puesto que no se identificaron ocupaciones del Período Tardío en los recintos, el resultado del trabajo de campo es de gran interés para comenzar a conocer una casa de fines 
Cerámicas de pasta fina

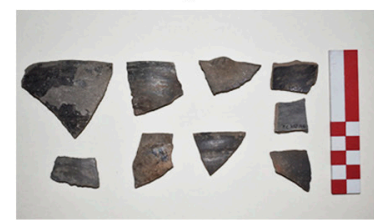

Gris pulido

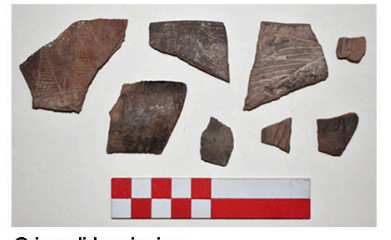

Gris pulido e inciso

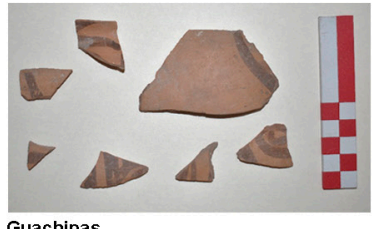

Guachipas

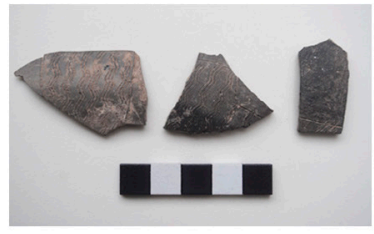

Gris pulido e inciso con peine

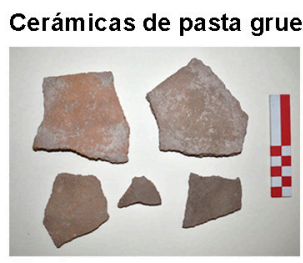

Alisadas

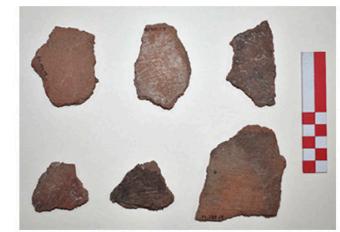

PEINADAS O MARLEADAS

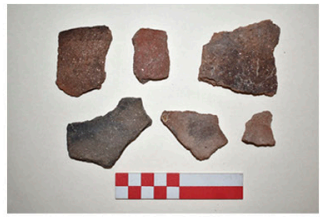

Alisadas, abundante mica (biotita)

Cerámicas de pasta media

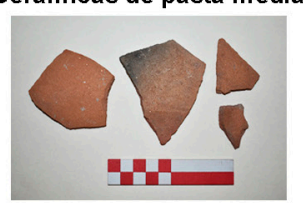

Alisadas

Figura 4. Ejemplos de fragmentos cerámicos recuperados en las excavaciones de la D28, Sector Conoide Norte, El Colorado.

Figure 4. Examples of ceramic fragments recovered in the excavations of D28, North Conoid Sector, El Colorado.

del primer milenio en el sur de Yocavil, una época poco conocida a partir del estudio de contextos domésticos. En síntesis, las excavaciones en la Detección 28 de El Colorado permitieron identificar una ocupación en la Estructura 65 y un mínimo de dos ocupaciones en la Estructura 66, cuya excavación aún no ha concluido. Se interpreta a la E65 como un espacio vinculado a la cocción de alimentos, debido a la presencia de un importante fogón con restos vegetales y óseos carbonizados, y se propone a la E66 como un área de habitación, funcionalmente vinculada a la primera. Considerando los resultados de un fechado radiocarbónico y los materiales cerámicos hallados en los pisos de los recintos (Figura 4) se estableció que la E65 y la ocupación superior de la E66 corresponden a los últimos siglos del primer milenio de la era. De acuerdo con diferentes propuestas de periodificación aplicadas a la zona, se rotula a esta época como Período Medio, Período de Integración Regional, Formativo Superior o fase Colalao (González 1979; Raffino 1991 [1988]; Scattolin 2010; Tartusi y Nuñez Regueiro 1993), pero más allá de la profusión de denominaciones, la misma se encuentra escasamente documentada en el valle de Yocavil.

Los ejemplos de ámbitos residenciales en Yocavil, para los cuales se cuenta con fechados por radiocarbono que los sitúan en momentos contemporáneos al uso de la Detección 28, exhiben diversidad en cuanto a las características constructivas, de emplazamiento y de escala. Los sitios Morro de las Espinillas, en el lado oriental del valle, y Morro del Fraile, en el interior de la Sierra del Cajón, poseen un patrón nucleado con predominio de recintos de planta ortogonal (Cigliano et al. 1960; Scattolin 2003; Nastri et al. 2005). Las instalaciones en la Mesada de Agua Salada o en Los Zazos, sitio El Remate, en el oriente, presentan recintos de planta circular que constituyen unidades residenciales dispersas entre áreas de producción agrícola (Lanzelotti y Spano 2015; Aschero y Ribotta 2007). El caso que presentamos aquí responde a una modalidad que no había sido reconocida para la época en el área de estudio, recintos contiguos, no adosados, de planta ortogonal, que conforman una pequeña unidad doméstica de tipo dispersa (Albeck 2000).
Las técnicas constructivas relevadas en esta unidad también resultan novedosas. Un elemento constructivo para el cual no se conocen antecedentes en el valle es la conjunción de cimientos de piedra dobles, columna de piedra y muros de barro. La construcción de columnas o pilares estructurales en piedra se identificó en instalaciones contemporáneas en otros ámbitos valliserranos, como los sitios Aguada del departamento de Ambato (Cruz 2006; Gordillo 2010), y en instalaciones más antiguas, como los sitios Alamito del Campo del Pucará (Núñez Regueiro 1998), ambos localizados hacia el sur de Yocavil, en la provincia de Catamarca.

Otro hallazgo de consideración para una reflexión sobre los signos de época son los abundantes fragmentos de cerámica ordinaria peinada en la ocupación superior de E66, asociados en un piso de ocupación a fragmentos de estilo ordinario alisado, gris pulido, gris pulido e inciso y Guachipas. Esta técnica de acabado de superficie es muy frecuente en las alfarerías ordinarias tardías en la zona (Palamarczuk 2008); la observación de fragmentos de vasijas ordinarias peinadas en la superficie del sector fue considerada por nosotros como posible indicador de una ocupación tardía. El hallazgo, debidamente contextualizado gracias a la excavación estratigráfica y en área extendida, muestra un antecedente que invita a revisar nuestro uso de esta categoría tecnológica, tomada de manera aislada, como un indicador cronológico inequívoco. Y constituye un caso con gran potencial para investigar el origen de las innovaciones técnicas -como el acabado de superficies en la alfarería- y la perduración y generalización de prácticas de producción en el tiempo.

La documentación de superposición de superficies de ocupación aporta un nuevo caso para estudiar las transformaciones en el modo de habitar el espacio doméstico, enhebrando los acontecimientos representados en la construcción y usos de la casa para la elaboración de una historia de los pobladores de El Colorado. Confiamos en que la finalización de las excavaciones, la obtención de nuevos fechados radiocarbónicos y el progreso en los análisis de los materiales recuperados, actualmente en 
curso, permitirán avanzar, partiendo desde un panorama descriptivo inicial, hacia una interpretación de un espacio habitacional de fines del primer milenio en Yocavil.

Buenos Aires, 12 de noviembre de 2019

\section{Agradecimientos}

Los autores expresamos nuestro agradecimiento a la Comunidad Originaria Ingamana, a la Intendencia de San José, a las Familias Villanueva y Chaile de Punta de Balasto, quienes propiciaron nuestros trabajos en la localidad. María Cecilia Fuertes de la Gerencia Química \& Instituto de Nanociencia y Nanotecnología (INN), Centro Atómico Constituyentes-CNEA CNEA, realizó los análisis por DRX. La investigación es financiada con los subsidios PICT 2014-0345, UBACYT 2018-2020 20020170100318BA, PIP 2014-2016 $11220130100178 C \mathrm{CO}$.

\section{Bibliografía}

Albeck, M. (2000). La vida agraria en los Andes del Sur. En: M. Tarragó (Dir.), Nueva Historia Argentina, Vol. 1, "Los pueblos originarios y la conquista" (pp. 187-228). Buenos Aires, Argentina: Editorial Sudamericana.

Aschero, C. y Ribotta, E. (2007). Usos del espacio, tiempo y funebria en El Remate (Los Zazos, Amaicha del Valle, Tucumán). En: P. Arenas, B. Manasse y E. Noli (Comps.), Paisajes y Procesos Sociales en Tafí. Una mirada interdisciplinaria desde el valle (Tucumán, Argentina) (pp. 79-94). Tucumán, Argentina: Universidad Nacional de Tucumán.

Braudel, F. (1970). La historia y las ciencias sociales. Madrid, España: Alianza Editorial.

Bronk Ramsey, C. (2017). OxCal program v4.3.2. Methods for summarizing radiocarbon datasets. Radiocarbon, 59 (2), 1809-1833.

Cigliano, E., De Gasperi, G. y Petruzzi, S. (1960). Pajanguillo. En: E. Cigliano (Ed.), Investigaciones Arqueológicas en el Valle de Santa María. Publicación Instituto de Antropología, (4), 43-51.

Cruz, P. (2006). Complejidad y heterogeneidad en los Andes meridionales durante el Período de Integración Regional (siglos IV-X d. C.). Nuevos datos acerca de la arqueología de la cuenca del río de Los Puestos (dpto. Ambato-Catamarca, Argentina). Bulletin de l'Institut Français d'Études Andines, 35 (2), 121-148.

González, A. R. (1979). Dinámica cultural en el NO argentino. Evolución e historia en las culturas del NO argentino. Antiquitas, (28-29), 1-15.

Gordillo, I. (2010). De piedra, tierra y madera. Arquitectura y prácticas sociales en la Iglesia de los Indios (Ambato, Catamarca). En: M. Albeck, C. Scattolin y A. Korstanje (Eds.), El hábitat prehispánico. Arqueología de la arquitectura y de la construcción del espacio organizad (pp. 155-185). Jujuy, Argentina: EDIUNJU.

Hogg, A., Hua, Q., Blackwell, P., Niu, M., Buck, C., Guilderson, T., Heaton, T., Palmer, J., Reimer, P., Reimer, R., Turney, C. y Zimmerman, S. (2013). SHCal13 Southern Hemisphere Calibration, 0-50,000 Years cal BP. Radiocarbon, 55 (4), 1889-1903.

Lanzelotti, S. y Spano, R. (2015). La multitemporalidad del paisaje en la Mesada del Agua Salada (Catamarca, Argentina). Arqueología, 21 (1), 47-71.

Maldonado M., Cordomí, A., Neder, L. y Sampietro Vattuone, M. (2012). Tiempo y espacio: el sitio «Talapazo» (Valle de Yocavil, Provincia de Tucumán). La Zaranda de Ideas, 8 (2), 101-117.

Nastri, J., Pratolongo, G., Reynoso, A. y Vargas, A. (2005). Arqueología en la Sierra del Cajón: poblados, corrales y pinturas. En: Actas del XV Congreso Nacional de Arqueología Argentina, Problemáticas de la arqueología contemporánea, (3), 715-728

Núñez Regueiro, V. (1998). Arqueología, historia y antropología de los sitios Alamito. Tucumán, Argentina: Ediciones Interdea.

Palamarczuk, V. (2008). Un análisis de la cerámica arqueológica de cuatro sitios en el bajo de Rincón Chico. En: M. Tarragó y L. González (Eds.), Investigaciones arqueológicas en Yocavil (pp. 20-80). Buenos Aires, Argentina: Proyecto Arqueológico Yocavil. Asociación de Amigos del Museo Etnográfico.

Palamarczuk, V. (2016). Investigaciones arqueológicas en El Colorado, sur de Yocavil, Catamarca, Argentina. Revista Andes, 27 (2), 1-28.

Raffino, R. (1991) (1988). Poblaciones Indígenas en la Argentina. Buenos Aires, Argentina: TEA.

Scattolin, C. (2003). Recursos arquitectónicos y estilos cerámicos en los siglos IX y X d.C. en el valle de Santa María (Catamarca, Argentina). En: P. Cornell y P. Stenborg (Eds.), Local, Regional, Global: prehistoria, protohistoria e historia en los Valles Calchaquíes. Etnologiska Studier, 46, 63-98.

Scattolin, C. (2010). La organización del hábitat precalchaquí (500 A.C. - 1000 D.C.). En: M. Albeck, C. Scattolin y A. Korstanje (Eds.), El hábitat prehispánico. Arqueología de la arquitectura y de la construcción del espacio organizad (pp. 13-51). Jujuy, Argentina: EDIUNJU.

Serrano, A. (1958). Manual de cerámica indígena. Córdoba, Argentina: Editorial Assandri.

Tartusi, M. y Nuñez Regueiro, V. (1993). Los centros ceremoniales del Noroeste Argentino. Publicaciones 5, Serie Ensayos (1), Tucumán, Argentina: Instituto de Arqueología de la Universidad Nacional de Tucumán. 